\title{
ON THE MAXIMUM NUMBER OF LIMIT CYCLES OF A CLASS OF GENERALIZED LIÉNARD DIFFERENTIAL SYSTEMS
}

\author{
JUSTINO ALAVEZ-RAMÍREZ ${ }^{1}$, GAMALIEL BLÉ ${ }^{1}$, \\ JORGE LÓPEZ-LÓPEZ ${ }^{1}$ AND JAUME LLIBRE ${ }^{2}$
}

\begin{abstract}
Applying the averaging theory of first, second and third order to one class generalized polynomial Liénard differential equations, we improve the known lower bounds for the maximum number of limit cycles that this class can exhibit.
\end{abstract}

\section{IntRoduCtion AND STATEMENT OF THE MAIN RESUltS}

The generalized polynomial Liénard differential equation

$$
\ddot{x}+f(x) \dot{x}+g(x)=0,
$$

or equivalently,

$$
\begin{aligned}
& \dot{x}=y, \\
& \dot{y}=-f(x) y-g(x),
\end{aligned}
$$

was introduced in [16]. Here the dot denotes derivative with respect to the independent variable $t$, and $f(x)$ and $g(x)$ are polynomials in the variable $x$ of degrees $n$ and $m$ respectively. For this subclass of polynomial vector fields we have a simplified version of the 16th Hilbert's problem, see [17] and [28].

In 1977 Lins, de Melo and Pugh [17] studied the classical polynomial Liénard differential equations (1) with $g(x)=x$ and stated the following conjecture: if $f(x)$ has degree $n \geq 1$ and $g(x)=x$, then (1) has at most $[n / 2]$ limit cycles. Here $[x]$ denotes the integer part function of $x \in \mathbb{R}$. They also proved the conjecture for $n=1,2$, and additionally they showed that there are systems (1) having at least $[n / 2]$ limit cycles. For $n \geq 5$ this conjecture is not true as it has been proved recently by Dumortier, Panazzolo and Roussarie in [6] and by De Maesschalck and Dumortier in [5]. More recently the conjecture has been proved for $n=3$ by Chengzhi Li and Llibre see $[13,14]$. In short the conjecture only remains open for $n=4$.

We note that a classical polynomial Liénard differential equation has a unique singular point. However it is possible for generalized polynomial Liénard differential equations to have more than one singular point.

Many of the results on the limit cycles of polynomial differential systems have been obtained by considering limit cycles which bifurcate from a single degenerate

1991 Mathematics Subject Classification. 34C29, 34C25.

Key words and phrases. Limit cycles, periodic orbits, Liénard differential equation, averaging theory.

This work is partially supported by grant CONACYT-58968. The fourth author is supported by the grants MCYT/FEDER MTM 2008-03437, Generalitat de Catalunya 2009SGR410 and ICREA Academia. 
singular point, that are so called small amplitud limit cycles, see [20]. We denote by $\hat{H}(m, n)$ the maximum number of small amplitude limit cycles for systems of the form (1). The values of $\hat{H}(m, n)$ give a lower bound for the maximum number $H(m, n)$ (i.e. the Hilbert number) of limit cycles that the differential equation (1) with $m$ and $n$ fixed can have. It is unknown the finitude of $H(m, n)$ for every positive integers $m$ and $n$. For more information about the Hilbert's 16th problem and related topics see [12] and [15].

Now we shall describe briefly the main results about the limit cicles on Liénard differential systems.

(i) In 1928 Liénard [16] proved that if $m=1$ and $F(x)=\int_{0}^{x} f(s) d s$ is a continuous odd function, which has a unique root at $x=a$ and is monotone increasing for $x \geq a$, then equation (1) has a unique limit cycle.

(ii) In 1973 Rychkov [27] proved that if $m=1$ and $F(x)=\int_{0}^{x} f(s) d s$ is an odd polynomial of degree five, then equation (1) has at most two limit cycles.

(iii) In 1977 Lins, de Melo and Pugh [17] proved that $H(1,1)=0$ and $H(1,2)=$ 1.

(iv) In 1990,1996, Dumortier, Li and Rousseau in [9] and [7] proved that $H(3,1)=$ 1.

(v) In 1998 Coppel [4] proved that $H(2,1)=1$.

(vi) In 1997 Dumortier and Chengzhi Li [8] proved that $H(2,2)=1$.

(vii) In 2010 Chengzhi $\mathrm{Li}$ and Llibre $[13,14]$ proved that $H(1,3)=1$.

Up to now and as far as we know only for the five cases (iii)-(vii) the Hilbert numbers $H(m, n)$ are determined.

Blows, Lloyd and Lynch, [1], [21] and [23] have used inductive arguments in order to prove the following results.

(I) If $g$ is odd then $\hat{H}(m, n)=[n / 2]$.

(II) If $f$ is even then $\hat{H}(m, n)=n$, whatever $g$ is.

(III) If $f$ is odd then $\hat{H}(m, 2 n+1)=[(m-2) / 2]+n$.

(IV) If $g(x)=x+g_{e}(x)$, where $g_{e}$ is even then $\hat{H}(2 m, 2)=m$.

Christopher and Lynch [3], [24], [25], [26] have developed a new algebraic method for determining the Liapunov quantities of system (1) and proved the following:

(V) $\hat{H}(m, 2)=[(2 m+1) / 3]$.

(VI) $\hat{H}(2, n)=[(2 n+1) / 3]$.

(VII) $\hat{H}(m, 3)=2[(3 m+2) / 8]$ for all $1<m \leq 50$.

(VIII) $\hat{H}(3, n)=2[(3 n+2) / 8]$ for all $1<m \leq 50$.

(IX) $\hat{H}(4, k)=\hat{H}(k, 4)$, for $k=6,7,8,9$ and $\hat{H}(5,6)=\hat{H}(6,5)$.

In 1998 Gasull and Torregrosa [10] obtained upper bounds for $\hat{H}(7,6), \hat{H}(6,7)$, $\hat{H}(7,7)$ and $\hat{H}(4,20)$.

In 2006 the values for $\hat{H}(m, n)=\hat{H}(n, m)$, for $n=4, m=10,11,12,13 ; n=5$, $m=6,7,8,9 ; n=6, m=5,6$ were given by Yu and Han in [30].

Llibre, Mereu and Teixeira [19] using the averaging theory studied the maximum number of limit cycles $\tilde{H}(m, n)$ which can bifurcate from the periodic orbits of a linear center perturbed inside the class of generalized polynomial Liénard differential 
equations of degrees $m$ and $n$ of the form

$$
\begin{aligned}
& \dot{x}=y, \\
& \dot{y}=-x-\sum_{k \geq 1} \varepsilon^{k}\left(f_{n}^{k}(x) y+g_{m}^{k}(x)\right),
\end{aligned}
$$

where for every $k$ the polynomials $g_{m}^{k}(x)$ and $f_{n}^{k}(x)$ have degree $m$ and $n$ respectively, and $\varepsilon$ is a small parameter, i.e. the maximal number of medium amplitude limit cycles which can bifurcate from the periodic orbits of the linear center $\dot{x}=y$, $\dot{y}=-x$, perturbed as in (2).

In fact in [19] the authors computed lower estimations of $\tilde{H}(m, n)$. More precisely they compute the maximum number of limit cycles $\tilde{H}_{k}(m, n)$ which bifurcate from the periodic orbits of the linear center $\dot{x}=y, \dot{y}=-x$, using the averaging theory of order $k$ for $k=1,2,3$. Of course $\tilde{H}_{k}(m, n) \leq \tilde{H}(m, n) \leq H(m, n)$. More precisely, the main result of [19] is: for $|\varepsilon|$ sufficiently small, the maximum number of medium limit cycles of the polynomial Liénard differential systems (2) bifurcating from the periodic orbits of the linear center $\dot{x}=y, \dot{y}=-x$, using the averaging theory

(a) of first order is $\tilde{H}_{1}(m, n)=\left[\frac{n}{2}\right]$;

(b) of second order is $\tilde{H}_{2}(m, n)=\max \left\{\left[\frac{n-1}{2}\right]+\left[\frac{m}{2}\right],\left[\frac{n}{2}\right]\right\}$; and

(c) of third order is $\tilde{H}_{3}(m, n)=\left[\frac{n+m-1}{2}\right]$.

Note that before the work [19] there were no lower estimations for $H(m, n)$ when

(a) $m=4$ and $n>13$, or $m>20$ and $n=4$,

(b) $m=5$ and $n>9$, or $m>9$ and $n=5$,

(c) $m=6$ and $n>7$, or $m>7$ and $n=6$,

(d) $m, n>7$.

After the results of [19] we will have lower estimations of $H(m, n)$ for all $m, n \geq 1$. It seems that the numbers $\hat{H}(m, n)$ can be symmetric with respect $m$ and $n$. Some studies is this direction are made in [22]. We remark that in general $\tilde{H}_{k}(m, n) \neq$ $\tilde{H}_{k}(n, m)$ for $k=1,2$, but $\tilde{H}_{3}(m, n)=\tilde{H}_{3}(n, m)$.

In this work using the averaging theory we study the maximum number of limit cycles $\tilde{H}(l, m, n)$ which can bifurcate from the periodic orbits of a linear center perturbed inside the class of generalized polynomial Liénard differential equations of degrees $l, m$ and $n$ of the form

$$
\begin{aligned}
& \dot{x}=y+\sum_{k \geq 1} \varepsilon^{k} h_{l}^{k}(x), \\
& \dot{y}=-x-\sum_{k \geq 1} \varepsilon^{k}\left(f_{n}^{k}(x) y+g_{m}^{k}(x)\right),
\end{aligned}
$$

where for every $k$ the polynomials $h_{l}^{k}(x), g_{m}^{k}(x)$ and $f_{n}^{k}(x)$ have degree $l, m$ and $n$ respectively, and $\varepsilon$ is a small parameter, i.e. the maximal number of medium amplitude limit cycles which can bifurcate from the periodic orbits of the linear center $\dot{x}=y, \dot{y}=-x$, perturbed as in (3). Of course the computation of $\tilde{H}(l, m, n)$ for the differential system (3) is another step in order to estimate the Hilbert number for this class of polynomial differential systems. 
Let $k$ be a positive integer. We define $E(k)$ as the largest even integer $\leq k$, and $O(k)$ as the largest odd integer $\leq k$. Our main result that improve the mentioned previous results is the following one.

Theorem 1. If for every $k=1,2$ the polynomials $h_{l}^{k}(x), g_{m}^{k}(x)$ and $f_{n}^{k}(x)$ have degree $l, m$ and $n$ respectively, with $l, m, n \geq 1$, then for $|\varepsilon|$ sufficiently small, the maximum number of medium limit cycles of the polynomial Liénard differential systems (3) bifurcating from the periodic orbits of the linear center $\dot{x}=y, \dot{y}=-x$, using the averaging theory

(a) of first order is

$$
\tilde{H}_{1}(l, m, n)=\left[\frac{\max \{O(l), O(n+1)\}-1}{2}\right]=\max \left\{\left[\frac{l-1}{2}\right],\left[\frac{n}{2}\right]\right\},
$$

(b) of second order is

$$
\tilde{H}_{2}(l, m, n)=\left[\frac{\max \{E(l)+E(m), O(n)+E(m)+1, O(l), O(n+1)\}-1}{2}\right],
$$

(c) of third order is

$$
\tilde{H}_{3}(l, m, n) \geq\left[\frac{\max \{O(m+n), E(l+m)-1\}-1}{2}\right], \text { and }
$$

(d) The three upper bounds for $\tilde{H}(l, m, n)$ given in statements (a), (b) and (c) for some values of $l, m$ and $n$ are reached. So they cannot be improved.

Of course if $H(l, m, n)$ is the Hilbert number for our polynomial Liénard differential systems $(3)$, then $\tilde{H}_{k}(l, m, n) \leq H(l, m, n)$ for $k=1,2,3$; i.e. the numbers $\tilde{H}_{k}(l, m, n)$ provide lower bounds for the Hilbert numbers of systems $(3)$.

In the proof of statement (c) we shall explain why in this statement we have an inequality instead of the equality of the previous two statements.

The rest of the paper is structured as follows. In section 2 we present a summary of the results on the averaging theory that we shall need in this paper. In sections $3,4,5$ and 6 we prove statements (a), (b), (c) and (d) of Theorem 1 respectively.

\section{The AVERAgIng THEORY OF FIRSt, SECOND AND THIRD ORDER}

The averaging theory of third order for studying specifically periodic orbits was developed in [2]. It is summarized as follows.

Consider the differential system

$$
\dot{x}(t)=\varepsilon F_{1}(t, x)+\varepsilon^{2} F_{2}(t, x)+\varepsilon^{3} F_{3}(t, x)+\varepsilon^{4} R(t, x, \varepsilon),
$$

where $F_{1}, F_{2}, F_{3}: \mathbb{R} \times D \rightarrow \mathbb{R}, R: \mathbb{R} \times D \times\left(-\varepsilon_{f}, \varepsilon_{f}\right) \rightarrow \mathbb{R}$ are continuous functions, $T$-periodic in the first variable, and $D$ is an open subset of $\mathbb{R}^{n}$. Assume that the following hypotheses (i) and (ii) hold.

(i) $F_{1}(t, \cdot) \in C^{2}(D), F_{2}(t, \cdot) \in C^{1}(D)$ for all $t \in \mathbb{R}, F_{1}, F_{2}, F_{3}, R, D_{x}^{2} F_{1}, D_{x} F_{2}$ are locally Lipschitz with respect to $x$, and $R$ is twice differentiable with respect to $\varepsilon$. 
We define $F_{k 0}: D \rightarrow \mathbb{R}$ for $k=1,2,3$ as

$$
\begin{gathered}
F_{10}(z)=\frac{1}{T} \int_{0}^{T} F_{1}(s, z) d s \\
F_{20}(z)=\frac{1}{T} \int_{0}^{T}\left[D_{z} F_{1}(s, z) \cdot y_{1}(s, z)+F_{2}(s, z)\right] d s, \\
F_{30}(z)=\frac{1}{T} \int_{0}^{T}\left[\frac{1}{2} y_{1}(s, z)^{\mathbf{T}} \frac{\partial^{2} F_{1}}{\partial z^{2}}(s, z) y_{1}(s, z)+\frac{1}{2} \frac{\partial F_{1}}{\partial z}(s, z) y_{2}(s, z)\right. \\
\left.+\frac{\partial F_{2}}{\partial z}(s, z) y_{1}(s, z)+F_{3}(s, z)\right] d s,
\end{gathered}
$$

where

$$
\begin{aligned}
& y_{1}(s, z)=\int_{0}^{s} F_{1}(t, z) d t \\
& y_{2}(s, z)=2 \int_{0}^{s}\left[\frac{\partial F_{1}}{\partial z}(t, z) \int_{0}^{t} F_{1}(r, z) d r+F_{2}(t, z)\right] d t .
\end{aligned}
$$

(ii) For $V \subset D$ an open and bounded set and for each $\varepsilon \in\left(-\varepsilon_{f}, \varepsilon_{f}\right) \backslash\{0\}$, there exists $a_{\varepsilon} \in V$ such that $F_{10}\left(a_{\varepsilon}\right)+\varepsilon F_{20}\left(a_{\varepsilon}\right)+\varepsilon^{2} F_{30}\left(a_{\varepsilon}\right)=0$ and $d_{B}\left(F_{10}+\varepsilon F_{20}+\varepsilon^{2} F_{30}, V, a_{\varepsilon}\right) \neq 0$.

Then for $|\varepsilon|>0$ sufficiently small there exists a $T$-periodic solution $\varphi(\cdot, \varepsilon)$ of the system such that $\varphi(0, \varepsilon)=a_{\varepsilon}$.

We remark that the 2 which appears in the beginning of the expression of $y_{2}(s, z)$ does not appear in [2]. This was a misprint in [2].

The expression $d_{B}\left(F_{10}+\varepsilon F_{20}+\varepsilon^{2} F_{30}, V, a_{\varepsilon}\right) \neq 0$ means that the Brouwer degree of the function $F_{10} \varepsilon F_{20}+\varepsilon^{2} F_{30}: V \rightarrow \mathbb{R}^{n}$ at the fixed point $a_{\varepsilon}$ is not zero. A sufficient condition for the inequality to be true is that the Jacobian of the function $F_{10}+\varepsilon F_{20}+\varepsilon^{2} F_{30}$ at $a_{\varepsilon}$ is not zero, if it is defined. In our applications since our differential Liénard systems are analytic, this Jacobian always is defined.

If $F_{10}$ is not identically zero, then the zeros of $F_{10}+\varepsilon F_{20}+\varepsilon^{2} F_{30}$ are mainly the zeros of $F_{10}$ for $\varepsilon$ sufficiently small. In this case the previous result provides the averaging theory of first order.

If $F_{10}$ is identically zero and $F_{20}$ is not identically zero, then the zeros of $F_{10}+$ $\varepsilon F_{20}+\varepsilon^{2} F_{30}$ are mainly the zeros of $F_{20}$ for $\varepsilon$ sufficiently small. In this case the previous result provides the averaging theory of second order.

If $F_{10}$ and $F_{20}$ are identically zero and $F_{30}$ is not identically zero, then the zeros of $F_{10}+\varepsilon F_{20}+\varepsilon^{2} F_{30}$ are mainly the zeros of $F_{30}$ for $\varepsilon$ sufficiently small. In this case the previous result provides the averaging theory of third order.

\section{Proof of statement (a) of Theorem 1}

We write

$$
f_{n}^{1}(x)=\sum_{i=0}^{n} a_{i} x^{i}, \quad g_{m}^{1}(x)=\sum_{i=0}^{m} b_{i} x^{i} \quad \text { and } \quad h_{l}^{1}(x)=\sum_{i=0}^{l} c_{i} x^{i} .
$$


Then in polar coordinates $(r, \theta)$ given by $x=r \cos \theta$ and $y=r \sin \theta$, the differential system (3) becomes

$$
\begin{aligned}
\dot{r}= & \varepsilon\left(\sum_{i=0}^{l} c_{i} r^{i} \cos ^{i+1} \theta-r \sin ^{2} \theta \sum_{i=0}^{n} a_{i} r^{i} \cos ^{i} \theta-\sin \theta \sum_{i=0}^{m} b_{i} r^{i} \cos ^{i} \theta\right)+O\left(\varepsilon^{2}\right), \\
\dot{\theta}= & -1-\frac{\varepsilon}{r}\left(r \cos \theta \sin \theta \sum_{i=0}^{n} a_{i} r^{i} \cos ^{i} \theta+\cos \theta \sum_{i=0}^{m} b_{i} r^{i} \cos ^{i} \theta+\sin \theta \sum_{i=0}^{l} c_{i} r^{i} \cos ^{i} \theta\right) \\
& +O\left(\varepsilon^{2}\right) .
\end{aligned}
$$

Taking $\theta$ as the new independent variable, this system writes

$$
\begin{aligned}
\frac{d r}{d \theta} & =-\varepsilon\left(\sum_{i=0}^{l} c_{i} r^{i} \cos ^{i+1} \theta-r \sin ^{2} \theta \sum_{i=0}^{n} a_{i} r^{i} \cos ^{i} \theta-\sin \theta \sum_{i=0}^{m} b_{i} r^{i} \cos ^{i} \theta\right)+O\left(\varepsilon^{2}\right) \\
& =\varepsilon F_{1}(\theta, r)+O\left(\varepsilon^{2}\right) .
\end{aligned}
$$

By using the notation introduced in section 2 we have that $F_{10}(r)=-\frac{1}{2 \pi} \int_{0}^{2 \pi}\left[\sum_{i=0}^{l} c_{i} r^{i} \cos ^{i+1} \theta-r \sin ^{2} \theta \sum_{i=0}^{n} a_{i} r^{i} \cos ^{i} \theta-\sin \theta \sum_{i=0}^{m} b_{i} r^{i} \cos ^{i} \theta\right] d \theta$

Since

$$
\int_{0}^{2 \pi} \cos ^{2 k+1} \theta d \theta=0, \quad \int_{0}^{2 \pi} \cos ^{2 k} \theta d \theta \neq 0, \quad \int_{0}^{2 \pi} \sin \theta \cos ^{k} \theta d \theta=0,
$$

for $k=0,1, \ldots$, we have that

$$
F_{10}(r)=-\frac{1}{2 \pi} \int_{0}^{2 \pi}\left[\sum_{\substack{i=2 \\ i \text { odd }}}^{l} c_{i} r^{i} \cos ^{i+1} \theta-r \sin ^{2} \theta \sum_{\substack{i=2 \\ i \text { even }}}^{n} a_{i} r^{i} \cos ^{i} \theta\right] d \theta
$$

We define

$$
M(l, n)= \begin{cases}\max \{l, n+1\} & \text { if } l \text { odd, } n \text { even } \\ \max \{l-1, n+1\} & \text { if } l \text { even, } n \text { even } \\ \max \{l, n\} & \text { if } l \text { odd, } n \text { odd } \\ \max \{l-1, n\} & \text { if } l \text { even, } n \text { odd }\end{cases}
$$

Then it is easy to check that

$$
M(l, n)=\max \{O(l), O(n+1)\},
$$

and that

$$
\left[\frac{M(l, n)-1}{2}\right]=\left[\frac{\max \{O(l), O(n+1)\}-1}{2}\right]=\max \left\{\left[\frac{l-1}{2}\right],\left[\frac{n}{2}\right]\right\} .
$$

Clearly we have that

with

$$
F_{10}(r)=\sum_{k=0}^{M(l, n)} \sigma_{k} r^{k}
$$

$$
\sigma_{k}=-\frac{1}{2 \pi} \int_{0}^{2 \pi}\left(c_{k} \cos ^{k+1} \theta-a_{k-1} \sin ^{2} \theta \cos ^{k-1} \theta\right) d \theta
$$

where $k \geq 1$ is an odd integer number, $a_{-1}=0, a_{k}=0$ if $k>n$ and $c_{k}=0$ if $k>l$. Of course $\sigma_{k}=0$ if $k$ is even. Since $F_{10}(r)$ is an odd function, it has at 
most $[(M(l, n)-1) / 2]$ simple positive real roots. From section 2 we obtain that for $|\varepsilon|$ sufficiently small, the maximum number of limit cycles of system (3) which can bifurcate from the periodic orbits of the linear center $\dot{x}=y, \dot{y}=-x$ using the averaging theory of first order is $[(M(l, n)-1) / 2]$. So statement (a) of Theorem 1 is proved.

\section{Proof of Statement (b) of Theorem 1}

In this section we consider the differential systems

$$
\begin{aligned}
& \dot{x}=y+\varepsilon h_{l}^{1}(x)+\varepsilon^{2} h_{l}^{2}(x)+O\left(\varepsilon^{3}\right), \\
& \dot{y}=-x-\varepsilon\left(f_{n}^{1}(x) y+g_{m}^{1}(x)\right)-\varepsilon^{2}\left(f_{n}^{2}(x) y+g_{m}^{2}(x)\right)+O\left(\varepsilon^{3}\right) .
\end{aligned}
$$

Taking polar coordinate this system becomes

$$
\begin{aligned}
& \dot{r}=\varepsilon \frac{x h_{l}^{1}(x)-y^{2} f_{n}^{1}(x)-y g_{m}^{1}(x)}{r}+\varepsilon^{2} \frac{x h_{l}^{2}(x)-y^{2} f_{n}^{2}(x)-y g_{m}^{2}(x)}{r}+O\left(\varepsilon^{3}\right), \\
& \dot{\theta}=-1-\varepsilon \frac{x y f_{n}^{1}(x)+x g_{m}^{1}(x)+y h_{l}^{1}(x)}{r^{2}}-\varepsilon^{2} \frac{x y f_{n}^{2}(x)+x g_{m}^{2}(x)+y h_{l}^{2}(x)}{r^{2}}+O\left(\varepsilon^{3}\right),
\end{aligned}
$$

where $x=r \cos \theta$ and $y=r \sin \theta$. This system is equivalent to

(6)

$$
\begin{aligned}
\frac{d r}{d \theta}= & -\varepsilon \frac{x h_{l}^{1}(x)-y^{2} f_{n}^{1}(x)-y g_{m}^{1}(x)}{r}-\varepsilon^{2}\left[\frac{x h_{l}^{2}(x)-y^{2} f_{n}^{2}(x)-y g_{m}^{2}(x)}{r}-\right. \\
& \left.\frac{\left(x h_{l}^{1}(x)-y^{2} f_{n}^{1}(x)-y g_{m}^{1}(x)\right)\left(x y f_{n}^{1}(x)+x g_{m}^{1}(x)+y h_{l}^{1}(x)\right)}{r^{3}}\right]+ \\
\varepsilon^{3}\left[\frac{\left(x h_{l}^{1}(x)-y^{2} f_{n}^{1}(x)-y g_{m}^{1}(x)\right)\left(x y f_{n}^{2}(x)+x g_{m}^{2}(x)+y h_{l}^{2}(x)\right)}{r^{3}}+\right. & \frac{\left(x h_{l}^{2}(x)-y^{2} f_{n}^{2}(x)-y g_{m}^{2}(x)\right)\left(x y f_{n}^{1}(x)+x g_{m}^{1}(x)+y h_{l}^{1}(x)\right)}{r^{3}}- \\
& \left.\frac{\left(x h_{l}^{1}(x)-y^{2} f_{n}^{1}(x)-y g_{m}^{1}(x)\right)\left(x y f_{n}^{1}(x)+x g_{m}^{1}(x)+y h_{l}^{1}(x)\right)^{2}}{r^{5}}\right]+O\left(\varepsilon^{4}\right) \\
= & \varepsilon F_{1}(\theta, r)+\varepsilon^{2} F_{2}(\theta, r)+\varepsilon^{3} F_{3}(\theta, r)+O\left(\varepsilon^{4}\right) .
\end{aligned}
$$

The explicit expression of $F_{1}(\theta, r)$ already has been done in section 3 . Now using the results stated in section 2 we shall apply the second order averaging theory to the previous differential equation, but for doing that we need that $F_{10}(r)=0$. Therefore, from (5) in what follows we must take

$$
c_{k}=a_{k-1}=0 \text { for all } k \text { odd. }
$$

From section 2 we must compute

$$
F_{20}(r)=\frac{1}{2 \pi} \int_{0}^{2 \pi}\left(D_{r} F_{1}(\theta, r) \int_{0}^{\theta} F_{1}(t, r) d t+F_{2}(\theta, r)\right) d \theta .
$$

Next we calculate the terms of this integral. First we have that

$$
\begin{aligned}
D_{r} F_{1}(\theta, r)= & -\sum_{\substack{i=2 \\
i \text { even }}}^{l} i c_{i} r^{i-1} \cos ^{i+1} \theta+\sum_{\substack{i=1 \\
i \text { odd }}}^{n}(i+1) a_{i} r^{i} \cos ^{i} \theta \sin ^{2} \theta \\
& +\sum_{i=1}^{m} i b_{i} r^{i-1} \cos ^{i} \theta \sin \theta
\end{aligned}
$$


Since

(9)

$$
\begin{aligned}
\int_{0}^{\theta} F_{1}(t, r) d t= & -\sum_{\substack{j=0 \\
j \text { even }}}^{l} c_{j} r^{j} \int_{0}^{\theta} \cos ^{j+1} t d t+\sum_{\substack{j=1 \\
j \text { odd }}}^{n} a_{j} r^{j+1} \int_{0}^{\theta} \cos ^{j} t \sin ^{2} t d t \\
& +\sum_{j=0}^{m} b_{j} r^{j} \int_{0}^{\theta} \cos ^{j} t \sin t d t \\
= & -\sum_{j=0}^{l} c_{j} r^{j} A_{j+1}(\theta)+\sum_{\substack{j=1 \\
j \text { odd }}}^{n} a_{j} r^{j+1}\left(A_{j}(\theta)-A_{j+2}(\theta)\right) \\
& +\sum_{j=0}^{m} \frac{1}{j+1} b_{j} r^{j}\left(1-\cos ^{j+1} \theta\right),
\end{aligned}
$$

where for $s$ odd we have

$$
\begin{aligned}
A_{s}(\theta)= & \int_{0}^{\theta} \cos ^{s} t d t \\
= & \sum_{\substack{k=1 \\
k \text { odd } \\
k-2}} \frac{(s-k) !}{s !} \frac{(s-k)^{2}(s-(k-2))^{2} \cdots(s-1)^{2}}{(s-k)^{2}} \sin \theta \cos ^{s-k} \theta \\
& +\frac{(s-1)^{2}(s-3)^{2} \cdots(2)^{2}}{s !} \sin \theta,
\end{aligned}
$$

see for more details [11].

From the nine main products of $D_{r} F_{1}(\theta, r) \int_{0}^{\theta} F_{1}(t, r) d t$ (see (8) and (9)), only the following four are not zero when we integrate them between 0 and $2 \pi$ :

$$
\begin{aligned}
& \sum_{\substack{i=2 \\
i \text { even }}}^{l} \sum_{\substack{j=0 \\
\text { even }}}^{m} \frac{i}{j+1} c_{i} b_{j} r^{i+j-1} \cos ^{i+j+2} \theta- \\
& \sum_{\substack{i=1 \\
i \text { odd } j=0}}^{n} \sum_{\substack{j=0 \\
\text { even }}}^{m} \frac{i+1}{j+1} a_{i} b_{j} r^{i+j} \cos ^{i+j+1} \theta \sin ^{2} \theta- \\
& \sum_{\substack{i=2 \\
i \text { even }}}^{m} \sum_{\substack{j=0 \\
\text { even }}}^{l} i b_{i} c_{j} r^{i+j-1} \cos ^{i} \theta \sin \theta A_{j+1}(\theta)+ \\
& \sum_{\substack{i=2 \\
i \text { even }}}^{m} \sum_{j=1}^{n} i b_{i} a_{j} r^{i+j} \cos ^{i} \theta \sin \theta\left(A_{j}(\theta)-A_{j+2}(\theta)\right) .
\end{aligned}
$$

Then the fourth sums of (10) are odd polynomial in the variable $r$ of degree $E(l)+$ $E(m)-1, O(n)+E(m), E(l)+E(m)-1$ and $O(n)+E(m)$, respectively. Therefore

$$
\frac{1}{2 \pi} \int_{0}^{2 \pi}\left(D_{r} F_{1}(\theta, r) \int_{0}^{\theta} F_{1}(t, r) d t\right) d \theta
$$


is an odd polynomial in the variable $r$ of degree $\max \{E(l)+E(m)-1, O(n)+E(m)\}$. Hence (11) can contribute at most with

$$
\left[\frac{\max \{E(l)+E(m)-1, O(n)+E(m)\}-1}{2}\right]
$$

simple positive real roots to the roots of $F_{20}(r)$.

Now we shall study the contribution of $\frac{1}{2 \pi} \int_{0}^{2 \pi} F_{2}(\theta, r) d \theta$ to $F_{20}(r)$. The first part

$$
\frac{x h_{l}^{2}(x)-y^{2} f_{n}^{2}(x)-y g_{m}^{2}(x)}{r}
$$

of $F_{2}(\theta, r)$ (see $\left.(6)\right)$, contributes at the roots of $F_{20}(r)$ exactly as the function $F_{1}(\theta, r)$ contributes to $F_{10}(r)$; i.e. it contributes at most with

$$
\left[\frac{\max \{O(l), O(n+1)\}-1}{2}\right]
$$

simple positive real roots to the roots of $F_{20}(r)$.

Finally we shall study the contribution of the second part

$$
\frac{\left(x h_{l}^{1}(x)-y^{2} f_{n}^{1}(x)-y g_{m}^{1}(x)\right)\left(x y f_{n}^{1}(x)+x g_{m}^{1}(x)+y h_{l}^{1}(x)\right)}{r^{3}}
$$

of $F_{2}(\theta, r)$ to $F_{20}(r)$, which can be written as

$$
\begin{aligned}
& \frac{1}{r}\left[\sum_{\substack{i=0 \\
i \text { even }}}^{l} c_{i} r^{i} \cos ^{i+1} \theta-\sum_{\substack{i=1 \\
i \text { odd }}}^{n} a_{i} r^{i+1} \cos ^{i} \theta \sin ^{2} \theta-\sum_{i=0}^{m} b_{i} r^{i} \cos ^{i} \theta \sin \theta\right] \\
& {\left[\sum_{\substack{j=1 \\
j \text { odd }}}^{n} a_{j} r^{j+1} \cos ^{j+1} \theta \sin \theta+\sum_{j=0}^{m} b_{j} r^{j} \cos ^{j+1} \theta+\sum_{\substack{j=0 \\
j \text { even }}}^{l} c_{j} r^{j} \cos ^{j} \theta \sin \theta\right] .}
\end{aligned}
$$

From the nine products between the different sums only four will not be zero after the integration with respect to $\theta$ between 0 and $2 \pi$, and two of these four are equal. 
So the terms of (16) which will contribute to $F_{20}(r)$ are

$$
\begin{gathered}
\frac{1}{r}\left[\sum_{\substack{i=0 \\
i \text { even }}}^{l} \sum_{j=0}^{m} c_{i} b_{j} r^{i+j} \cos ^{i+j+2} \theta-2 \sum_{\substack{i=1 \\
i \text { odd }}}^{n} \sum_{j=0}^{m} a_{i} b_{j} r^{i+j+1} \cos ^{i+j+1} \theta \sin ^{2} \theta\right. \\
\left.-\sum_{\substack{i=0 \\
i \text { even }}}^{m} \sum_{j=0}^{l} b_{i} c_{j} r^{i+j} \cos ^{i+j} \theta \sin ^{2} \theta\right]= \\
\frac{1}{r}\left[\sum_{\substack{i=0 \\
i \text { even }}}^{l} \sum_{j=0}^{m} c_{i} b_{j} r^{i+j} \cos ^{i+j} \theta\left(\cos ^{2} \theta-\sin ^{2} \theta\right)\right. \\
\left.-2 \sum_{\substack{i=1 \\
i \text { odd }}}^{n} \sum_{j=0}^{m} a_{i} b_{j} r^{i+j+1} \cos ^{i+j+1} \theta \sin ^{2} \theta\right] .
\end{gathered}
$$

We note that the integral between 0 and $2 \pi$ with respect to $\theta$ of the term $i=j=0$ of the first sum of this last expression is zero. So the result of integrate between 0 and $2 \pi$ with respect to $\theta$ this last expression is an odd polynomial in the variable $r$ of degree $\max \{E(l)+E(m), O(n)+E(m)+1\}$. Consequently the contribution of (15) to the zeros of $F_{20}(r)$ is at most with

$$
\left[\frac{\max \{E(l)+E(m), O(n)+E(m)+1\}-1}{2}\right]
$$

simple positive real roots.

In short from (12), (14) and (18), we have that the polynomial $F_{20}(r)$ has at most

$$
\left[\frac{\max \{E(l)+E(m), O(n)+E(m)+1, O(l), O(n+1)\}-1}{2}\right]
$$

simple positive real roots. So, from the results of section 2 statement (b) of Theorem 1 is proved.

\section{Proof of statement $(c)$ of Theorem 1}

Since in this section we must repeat arguments that were used in the previous two sections, the long proof of this section is less detailed than the previous ones.

In this section we consider the differential systems (6). We write

$$
f_{n}^{2}(x)=\sum_{i=0}^{n} \hat{a}_{i} x^{i}, \quad g_{m}^{2}(x)=\sum_{i=0}^{m} \hat{b}_{i} x^{i} \quad \text { and } \quad h_{l}^{2}(x)=\sum_{i=0}^{l} \hat{c}_{i} x^{i} .
$$

We have obtained explicit expressions of $F_{1}(\theta, r)$ and $F_{2}(\theta, r)$ in the last sections. Now using the results stated in section 2, we shall apply the third order averaging theory to the previous differential equation, but for doing that we must assume that $F_{20}(r)=F_{10}(r) \equiv 0$. Therefore, from (5) and (13) we have that

$$
c_{k}=\hat{c}_{k}=a_{k-1}=\hat{a}_{k-1}=0 \text { for all } k \text { odd }
$$


and from (10) and (17) we get that if

$$
\text { either } f_{n}^{1}(x)=h_{l}^{1}(x) \equiv 0, \quad \text { or } \quad g_{m}^{1}(x) \equiv 0,
$$

then $F_{20}(r) \equiv 0$. These last two conditions are sufficient in order that $F_{20}(r) \equiv 0$, but they are not necessary. This explains the inequality of statement (c) instead of the equalities of the statements (a) and (b). But in any case we will show in the proof of statement $(\mathrm{d})$ that the inequality becomes equality for some particular Liénard differential systems. Note that $f_{n}^{1}(x)=h_{l}^{1}(x) \equiv 0$ forces that all the $a_{i}$ 's and $c_{i}$ 's are zero, while $g_{m}^{1}(x) \equiv 0$ implies that all the $b_{i}$ 's are zero.

5.1. Case $f_{n}^{1}(x)=h_{l}^{1}(x) \equiv 0$. In this part we shall apply the third order averaging theory to equation (6), considering $f_{n}^{1}(x)=h_{l}^{1}(x) \equiv 0$ and condition (19). From section 2 we must calculate

$$
\begin{aligned}
F_{30}(z)= & \frac{1}{T} \int_{0}^{T}\left[\frac{1}{2} y_{1}(s, z)^{\mathbf{T}} \frac{\partial^{2} F_{1}}{\partial z^{2}}(s, z) y_{1}(s, z)+\frac{1}{2} \frac{\partial F_{1}}{\partial z}(s, z) y_{2}(s, z)\right. \\
& \left.+\frac{\partial F_{2}}{\partial z}(s, z) y_{1}(s, z)+F_{3}(s, z)\right] d s
\end{aligned}
$$

where

$$
\begin{aligned}
y_{2}(t, r)= & 2 \sum_{i=1}^{m} \sum_{j=1}^{m} \frac{i}{j+1} b_{i} b_{j} r^{i+j-1}\left[\frac{1-\cos ^{i+1} t}{i+1}+\frac{\cos ^{i+j+2} t-1}{i+j+2}\right]- \\
& 2 \sum_{\substack{i=0 \\
i \text { even }}}^{l} \hat{c}_{i} r^{i} A_{i+1}(t)+2 \sum_{\substack{i=0 \\
i \text { odd } \\
m}}^{n} \hat{a}_{i} r^{i+1}\left[A_{i+1}(t)-A_{i+2}(t)\right]+ \\
& 2 \sum_{i=0}^{\hat{b}_{i}} \frac{m}{i+1}\left(1-\cos ^{i+1} t\right)-2 \sum_{i=1}^{m} \sum_{j=1}^{m} \frac{b_{i} b_{j}}{i+j+2} r^{i+j-1}\left(1-\cos ^{i+j+2} t\right) .
\end{aligned}
$$

In this case, from (8), we have

$$
\frac{\partial^{2} F_{1}}{\partial r^{2}}(t, r)=\sum_{i=2}^{m} i(i-1) b_{i} r^{i-2} \cos ^{i} t \sin t
$$

Thus all terms of the sums obtained from

$$
\left(y_{1}(t, r)\right)^{2} \frac{\partial^{2} F_{1}}{\partial r^{2}}(t, r)
$$

have $\sin t$. Therefore the integral between 0 and $2 \pi$ with respect $t$ is zero and this first part does not contribute to $F_{30}$. From (8) we have that

$$
\frac{\partial F_{1}}{\partial r}(t, r) y_{2}(t, r)=\left(\sum_{i=1}^{m} i b_{i} r^{i-1} \cos ^{i} t \sin t\right) y_{2}(t, r) \text {. }
$$

In this expression the integral between 0 and $2 \pi$ with respect to $t$ is not zero only in the sums having terms $A_{i}(t)$ with $i$ odd. Thus we obtain that this part contribute to $F_{30}(r)$ with an odd polynomial in the variable $r$ of degree 


$$
\max \{E(l)+E(m)-1, O(n)+E(m)\} .
$$

From (8) and (21) we obtain that $\frac{\partial F_{2}}{\partial r}(t, r) y_{1}(t, r)$ contribute to $F_{30}(r)$ with an odd polynomial in the variable $r$ of degree

$$
\max \{E(l+m)-1, O(m+n)\}
$$

Finally, since

$$
\begin{aligned}
F_{3}(t, r)= & -2 \sum_{i=1}^{m} \sum_{j=0}^{n} b_{i} \hat{a}_{j} r^{i+j} \cos ^{i+j+1} t \sin ^{2} t-2 \sum_{i=1}^{m} \sum_{j=0}^{m} b_{i} \hat{b}_{j} r^{i+j-1} \cos ^{i+j+1} t \sin t+ \\
& 2 \sum_{i=1}^{m} \sum_{j=0}^{l} b_{i} \hat{c}_{j} r^{i+j-1} \cos ^{i+j+2} t-\sum_{i=1}^{m} \sum_{j=0}^{l} b_{i} \hat{c}_{j} r^{i+j-1} \cos ^{i+j} t+ \\
& \frac{1}{r^{2}}\left(\sum_{i=1}^{m} b_{i} r^{i} \cos ^{i} t\right)^{3} \cos ^{2} t \sin t
\end{aligned}
$$

only the first, third and fourth sums contributing to $F_{30}(r)$ with an odd polynomial in the variable $r$ of degree

$$
\max \{O(m+n), E(m+l)-1\} .
$$

Thus from $(22),(23)$ and $(24)$ we have that the polinomial $F_{30}(r)$ has at most

$$
\left[\frac{\max \{O(m+n), E(m+l)-1\}-1}{2}\right]
$$

simple positive real roots.

5.2. Case $g_{m}^{1}(x) \equiv 0$. From section 2 the expression of $F_{30}$ includes the terms

$$
\begin{gathered}
\frac{\partial^{2} F_{1}}{\partial z^{2}}(s, z)\left(y_{1}(s, z)\right)^{2}, \\
\frac{\partial F_{1}}{\partial z}(s, z) y_{2}(s, z), \\
\frac{\partial F_{2}}{\partial z}(s, z) y_{1}(s, z) \text { and } F_{3}(s, z),
\end{gathered}
$$

that we must integrate between 0 and $2 \pi$.

From (8) we have in this case

$$
\frac{\partial^{2} F_{1}}{\partial r^{2}}(t, r)=-\sum_{\substack{i=2 \\ i \text { even }}}^{l} i(i-1) c_{i} r^{i-2} \cos ^{i+1} t+\sum_{\substack{i=1 \\ i \text { odd }}}^{n} i(i+1) a_{i} r^{i-1} \cos ^{i} t \sin ^{2} t .
$$


From (9) we obtain that

$$
\begin{aligned}
& y_{1}(t, r)^{2}=\sum_{\substack{i=0 \\
i \text { even }}}^{l} \sum_{\substack{j=0 \\
\text { even }}}^{l} c_{i} c_{j} r^{i+j} A_{i+1}(t) A_{j+1}(t)- \\
& 2 \sum_{\substack{i=0 \\
i \text { even }}}^{l} \sum_{\substack{j=1 \\
j \text { odd }}}^{n} c_{i} a_{j} r^{i+j+1} A_{i+1}(t)\left(A_{j}(t)-A_{j+2}(t)\right)+ \\
& \sum_{\substack{i=1 \\
i \text { odd }}}^{n} \sum_{\substack{j=1 \\
j \text { odd }}}^{n} a_{i} a_{j} r^{i+j+2}\left(A_{i}(t)-A_{i+2}(t)\right)\left(A_{j}(t)-A_{j+2}(t)\right) .
\end{aligned}
$$

Therefore

$$
\frac{\partial^{2} F_{1}}{\partial z^{2}}(s, z)\left(y_{1}(s, z)\right)^{2}
$$

does not contribute to $F_{30}$ because all terms of (26) have $\cos t$ to odd power. Now we shall study the contribution of

$$
\frac{\partial F_{1}}{\partial z}(s, z) y_{2}(s, z)
$$

We start calculating

$$
y_{2}(s, z)=\int_{0}^{s}\left[\frac{\partial F_{1}}{\partial z}(t, z) \int_{0}^{t} F_{1}(r, z) d r+F_{2}(t, z)\right] d t .
$$

From (8) and (9), we obtain in this case that

$$
\frac{\partial F_{1}}{\partial r}(t, r) \int_{0}^{t} F_{1}(s, r) d s=\left(\begin{array}{l}
-\sum_{\substack{i=2 \\
i \text { even }}}^{l} i c_{i} r^{i-1} \cos ^{i+1} t+\sum_{\substack{i=1 \\
i \text { odd }}}^{n}(i+1) a_{i} r^{i} \cos ^{i} t \sin ^{2} t \\
-\sum_{\substack{i=0 \\
i \text { even }}}^{l} c_{i} r^{i} A_{i+1}(t)+\sum_{\substack{i=1 \\
i \text { odd }}}^{n} a_{i} r^{i+1}\left(A_{i}(t)-A_{i+2}(t)\right)
\end{array}\right) .
$$

Since $A_{i}(t)$ can be written as

$$
A_{i}(t)=\sum_{\substack{j=0 \\ j \text { even }}}^{i-1} \alpha_{j}(i) \cos ^{j} t \sin t
$$


we obtain that

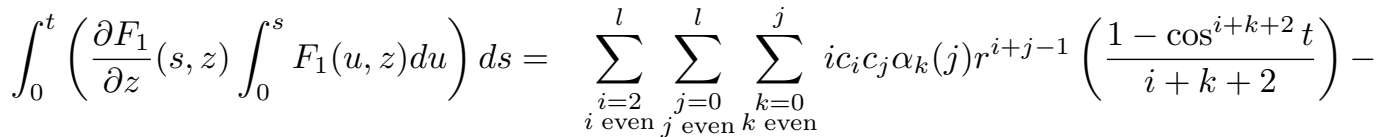

$$
\begin{aligned}
& \sum_{\substack{i=2 \\
i \text { even }}}^{l} \sum_{\substack{j=1 \\
j \text { odd }}}^{n} \sum_{\substack{k=0 \\
\text { even }}}^{j-1} i c_{i} a_{j} \alpha_{k}(j) r^{i+j}\left(\frac{1-\cos ^{i+k+2} t}{i+k+2}\right)+ \\
& \sum_{\substack{i=2 \\
i \text { even }}}^{l} \sum_{\substack{j=1 \\
j \text { odd }}}^{n} \sum_{\substack{k=0 \\
k \text { even }}}^{j+1} i c_{i} a_{j} \alpha_{k}(j) r^{i+j}\left(\frac{1-\cos ^{i+k+2} t}{i+k+2}\right)- \\
& \sum_{\substack{i=1 \\
i \text { odd }}}^{n} \sum_{\substack{j=0 \\
\text { even }}}^{l} \sum_{\substack{k=0 \\
\text { even }}}^{j}(i+1) a_{i} c_{j} \alpha_{k}(j) r^{i+j} \\
& {\left[\left(\frac{1-\cos ^{i+k+1} t}{i+k+1}\right)-\left(\frac{1-\cos ^{i+k+3} t}{i+k+3}\right)\right]-} \\
& \sum_{\substack{i=1 \\
i \text { odd }}}^{n} \sum_{\substack{j=1 \\
j \text { odd }}}^{n} \sum_{\substack{k=0 \\
k \text { even }}}^{j-1}(i+1) a_{i} a_{j} \alpha_{k}(j) r^{i+j+1} \text {. } \\
& {\left[\left(\frac{1-\cos ^{i+k+1} t}{i+k+1}\right)-\left(\frac{1-\cos ^{i+k+3} t}{i+k+3}\right)\right]} \\
& \sum_{\substack{i=1 \\
i \text { odd }}}^{n} \sum_{\substack{j=1 \\
\text { odd } \\
k \text { even }}}^{n} \sum_{\substack{k=0 \\
\text { even }}}^{j+1}(i+1) a_{i} a_{j} \alpha_{k}(j) r^{i+j+1} \text {. } \\
& {\left[\left(\frac{1-\cos ^{i+k+1} t}{i+k+1}\right)-\left(\frac{1-\cos ^{i+k+3} t}{i+k+3}\right)\right] .}
\end{aligned}
$$

The other term of $y_{2}(t, r)$ is

$$
\begin{aligned}
\int_{0}^{t} F_{2}(s, r) d s= & -\sum_{\substack{i=0 \\
i \text { even }}}^{l} \hat{c}_{i} r^{i} A_{i+1}(t)+\sum_{\substack{i=1 \\
i \text { odd }}}^{n} \hat{a}_{i} r^{i+1}\left(A_{i}(t)-A_{i+2}(t)\right)+ \\
& \sum_{i=0}^{m} \hat{b}_{i} r^{i}\left(\frac{1-\cos ^{i+1} t}{i+1}\right)+2 \sum_{\substack{i=2 \\
i \text { even } \\
j \text { odd }}}^{l} \sum_{j=1}^{n} c_{i} a_{j} r^{i+j}\left(\frac{1-\cos ^{i+j+3} t}{i+j+3}\right)- \\
& \sum_{i=1}^{n} \sum_{j=2}^{l} a_{i} c_{j} r^{i+j}\left(\frac{1-\cos ^{i+j+1} t}{i+j+1}\right)+ \\
& \sum_{\substack{i=2 \\
i \text { odd } \\
j}}^{l} \sum_{j=2}^{l} c_{j} c_{i} c_{j} r^{i+j-1}\left(\frac{1-\cos ^{i+j+2} t}{i+j+2}\right)- \\
& \sum_{\substack{i=1 \\
i \text { odd }}}^{n} \sum_{j=1}^{n} a_{j} a_{i} a_{j} r^{i+j+1}\left[\left(\frac{1-\cos ^{i+j+2} t}{i+j+2}\right)-\left(\frac{1-\cos ^{i+j+4} t}{i+j+4}\right)\right] .
\end{aligned}
$$


From the above we determine that the first part of $y_{2}(t, r)$ does not contribute to $F_{30}(r)$ and the second part only contribute with the terms

$$
\sum_{\substack{i=2 \\ i \text { even }}}^{l} \sum_{j=0}^{m} \frac{i}{j+1} c_{i} \hat{b}_{j} r^{i+j-1} \cos ^{i+j+2} t,
$$

and

$$
\sum_{\substack{i=1 \\ i \text { odd }}}^{n} \sum_{j=0}^{m} \frac{(i+1)}{j+1} a_{i} \hat{b}_{j} r^{i+j}\left(\cos ^{i+j+1} t-\cos ^{i+j+3} t\right) .
$$

Thus the result of integrating both terms with respect to $t$ between 0 and $2 \pi$ is an odd polynomial in the variable $r$ of degree

$$
\max \{E(l)+E(m)-1, O(n)+E(m)\} .
$$

Now we shall study the contribution of term,

From (6) we obtain that

$$
\frac{\partial F_{2}}{\partial z}(s, z) y_{1}(s, z) \text {. }
$$

$$
\begin{aligned}
& \frac{\partial F_{2}}{\partial r}(t, r)=-\sum_{\substack{i=2 \\
i \text { even } \\
m}}^{l} i \hat{c}_{i} r^{i-1} \cos ^{i+1} t+\sum_{\substack{i=1 \\
i \text { odd }}}^{n}(i+1) \hat{a}_{i} r^{i}\left(1-\cos ^{2} t\right) \cos ^{i} t+ \\
& \sum_{i=1}^{l} i \hat{b}_{i} r^{i-1} \cos ^{i} t \sin t+2 \sum_{\substack{i=2 \\
i \text { even }}}^{l} \sum_{j=1}^{n}(i+j) c_{i} a_{j} r^{i+j-1} \cos ^{i+j+2} t \sin t- \\
& \sum_{\substack{i=1 \\
i \text { odd }}}^{n} \sum_{j=2}^{l}(i+j) a_{i} c_{j} r^{i+j-1} \cos ^{i+j} t \sin t+ \\
& \sum_{\substack{i=2 \\
i \text { even }}}^{l} \sum_{j=2}^{l}(i+j-1) c_{i} c_{j} r^{i+j-2} \cos ^{i+j+1} t \sin t- \\
& \sum_{\substack{i=1 \\
i \text { odd }}}^{n} \sum_{j=1}^{n}(i+j \text { odd } \\
&
\end{aligned}
$$

From above we determine that the only terms of $\frac{\partial F_{2}}{\partial z}(s, z) y_{1}(s, z)$ contributing to $F_{30}(t)$ are

$$
\sum_{\substack{i=0 \\ i \text { even }}}^{l} \sum_{j=0}^{m} j c_{i} \hat{b}_{j} r^{i+j-1} \cos ^{j} t \sin t A_{i+1}(t),
$$

and

$$
\sum_{\substack{i=1 \\ i \text { odd }}}^{n} \sum_{j=1}^{m} j a_{i} \hat{b}_{j} r^{i+j} \cos ^{j} t \sin t\left(A_{i}(t)-A_{i+2}(t)\right) .
$$

Thus the result of integrating both sums with respect to $t$ is an odd polynomial in the variable $r$ of degree 


$$
\max \{E(l)+E(m)-1, O(n)+E(m)\} .
$$

Finally we shall study the contribution of $F_{3}(t, r)$ to $F_{30}(r)$. From the eighteen terms of $F_{3}(t, r)$, only four will not be zero after integration with respect to $t$ between 0 and $2 \pi$, and two of these four are equal. Thus the terms of $F_{3}(t, r)$ which contribute to $F_{30}(r)$ are

$$
\begin{gathered}
\sum_{\substack{i=2 \\
i \text { even }}}^{l} \sum_{j=0}^{m} c_{i} \hat{b}_{j} r^{i+j-1} \cos ^{i+j+2} t, \\
\sum_{\substack{i=1 \\
i \text { odd }}}^{n} \sum_{j=0}^{m} a_{i} \hat{b}_{j} r^{i+j} \cos ^{i+j+1} t\left(1-\cos ^{2} t\right),
\end{gathered}
$$

and

$$
\sum_{\substack{i=2 \\ i \text { even }}}^{l} \sum_{j=0}^{m} c_{i} \hat{b}_{j} r^{i+j-1} \cos ^{i+j} t\left(1-\cos ^{2} t\right) .
$$

Thus the result of integrating between 0 and $2 \pi$ these three terms is an odd polynomial in the variable $r$ of degree

$$
\max \{E(l)+E(m)-1, O(n)+E(m)\} .
$$

From (27), (28) and (29), we have that the polynomial $F_{30}(r)$ has at most

$$
\left[\frac{\max \{E(l)+E(m)-1, O(n)+E(m)\}-1}{2}\right]
$$

simple positive real roots. Thus putting together the results of (25) and (30), taking into account that

$$
\left[\frac{O(m+n)-1}{2}\right]=\left[\frac{n+m-1}{2}\right] \text { and } \quad\left[\frac{O(n)+E(m)-1}{2}\right] \leq\left[\frac{n+m-1}{2}\right],
$$

and by the results of section 2 statement (c) of Theorem 1 is proved.

\section{Proof of Statement $(d)$ of Theorem 1}

First we need to prove that under the assumptions of statement (a) of Theorem 1 there are Liénard differential systems $(3)$ such that $\tilde{H}_{1}(l, m, n)$ is equal to either $[n / 2]$, or $[(l-1) / 2]$. As we mentioned in the introduction Lins, de Melo and Pugh showed that there are Liénard differential systems (3) for which $\tilde{H}_{1}(l, m, n)=[n / 2]$. Now we shall provide a system for which $\tilde{H}_{1}(l, m, n)=[(l-1) / 2]$. Indeed we consider the Liénard differential system

$$
\begin{aligned}
& \dot{x}=y+\varepsilon\left(4 x^{3}-3 x\right), \\
& \dot{y}=-x,
\end{aligned}
$$

with $l=3$ and $m=n=0$. An easy computation shows that $F_{10}(r)=3 r\left(r^{2}-1\right) / 2$. So from the periodic orbit of radius 1 of the linear center $\dot{x}=y, \dot{y}=-x$, it bifurcates one limit cycle. Consequently for system $(32)$ we have that $\tilde{H}_{1}(3,0,0)=$ $[(3-1) / 2]=1$. Hence the two values whose maximum is $\tilde{H}_{1}(l, m, n)$ in statement (a) of Theorem 1 are reached for convenient Liénard differential systems (3). 
Now we shall show that under the assumptions of statement (b) of Theorem 1 there are Liénard differential systems $(3)$ such that $\tilde{H}_{2}(l, m, n)$ is equal to

$$
\text { either }\left[\frac{O(n)+E(m)}{2}\right] \text {, or }\left[\frac{O(n+1)-1}{2}\right] \text {, or }\left[\frac{E(l)+E(m)-1}{2}\right] \text {. }
$$

Since

$$
\left[\frac{O(n)+E(m)}{2}\right]=\left[\frac{n-1}{2}\right]+\left[\frac{m}{2}\right], \text { and }\left[\frac{O(n+1)-1}{2}\right]=\left[\frac{n}{2}\right],
$$

the existence of Liénard differential systems (3) satisfying that

$$
\tilde{H}_{2}(l, m, n)=\left[\frac{O(n)+E(m)}{2}\right], \text { or } \tilde{H}_{2}(l, m, n)=\left[\frac{O(n+1)-1}{2}\right],
$$

follows from Theorem 1 and its proof of [19]. Now we shall present a system for which $\tilde{H}_{2}(l, m, n)=[(E(l)+E(m)-1) / 2]$. We consider the Liénard differential system

$$
\begin{aligned}
& \dot{x}=y+\varepsilon 2 x^{2}+\varepsilon^{2} x, \\
& \dot{y}=-x-\varepsilon x^{2},
\end{aligned}
$$

with $l=m=2$ and $n=0$. It is not difficult to check that $F_{10}(r) \equiv 0$ and $F_{20}(r)=r\left(r^{2}-1\right) / 2$. Therefore from the periodic orbit of radius 1 of the linear center $\dot{x}=y, \dot{y}=-x$, it bifurcates one limit cycle. Consequently for system (33) we have that $\tilde{H}_{2}(2,2,0)=[(E(2)+E(2)-1) / 2]=1$. Hence the three values whose maximum is $\tilde{H}_{2}(l, m, n)$ in statement $(b)$ of Theorem 1 are reached for convenient Liénard differential systems (3).

Finally we must prove that under the assumptions of statement (c) of Theorem 1 there are Liénard differential systems $(3)$ such that $\tilde{H}_{3}(l, m, n)$ is equal to

$$
\text { either }\left[\frac{O(m+n)-1}{2}\right] \text {, or }\left[\frac{E(l+m)-1}{2}\right] \text {. }
$$

From (31) we get that

$$
\left[\frac{O(m+n)-1}{2}\right]=\left[\frac{n+m-1}{2}\right]
$$

therefore the existence of Liénard differential systems (3) satisfying that

$$
\tilde{H}_{3}(l, m, n)=\left[\frac{O(m+n)-1}{2}\right],
$$

follows from Theorem 1 and its proof of [19]. Now we shall give a system for which $\tilde{H}_{3}(l, m, n)=[(E(l+m)-1) / 2]$. We consider the Liénard differential system

$$
\begin{aligned}
& \dot{x}=y+\varepsilon 4 x^{2}+\varepsilon^{3} 7 x, \\
& \dot{y}=-x-\varepsilon^{2} 6 x^{2},
\end{aligned}
$$

with $l=m=2$ and $n=0$. A tedious but easy computation shows that $F_{10}(r)=$ $F_{20}(r) \equiv 0$ and $F_{30}(r)=7 r\left(r^{2}-1\right) / 2$. Therefore from the periodic orbit of radius 1 of the linear center $\dot{x}=y, \dot{y}=-x$, it bifurcates one limit cycle. Consequently for system $(34)$ we have that $\left.\tilde{H}_{3}(2,2,0)=[O(2+2)-1) / 2\right]=1$. Hence the two values whose maximum provides a lower bound for $\tilde{H}_{3}(l, m, n)$ in statement $(c)$ of Theorem 1 are reached and coincides with maximum number of limit cycles bifurcating from 
the periodic orbits of the linear center for convenient Liénard differential systems

(3). In short statement (d) Theorem 1 is proved.

\section{REFERENCES}

[1] T.R. Blows AND N. G. Lloyd, The number of small-amplitude limit cycles of Liénard equations, Math. Proc. Camb. Phil. Soc. 95 (1984), 359-366.

[2] A. Buic̆ AND J. LliBRe, Averaging methods for finding periodic orbits via Brouwer degree, Bull. Sci. Math. 128 (2004), 7-22.

[3] C.J. Christopher And S. Lynch, Small-amplitude limti cycle bifurcations for Liénard systems with quadratic or cubic dapimg or restoring forces, Nonlinearity 12 (1999), 1099-1112.

[4] W. A. Coppel, Some quadratic systems with at most one limit cycles, Dynamics Reported, Vol.2,Wiley, New York, 1998, pp. 61-68.

[5] P. De Maesschalck and F. Dumortier, Classical Liénard equation of degree $n \geq 6$ can have $\left[\frac{n-1}{2}\right]+2$ limit cycles, preprint, 2010.

[6] F. Dumortier, D. Panazzolo and R. Roussarie, More limit cycles than expected in Liénard systems, Proc. Amer. Math. Soc. 135 (2007), 1895-1904.

[7] F. Dumortier AND C. LI, On the uniqueness of limit cycles surrounding one or more singularities for Liénard equations, Nonlinearity 9 (1996), 1489-1500.

[8] F. Dumortier And C. Li, Quadratic Liénard equations with quadratic damping, J. Diff. Eqs. 139 (1997), 41-59.

[9] F. Dumortier and C. Rousseau, Cubic Liénard equations with linear dapimg, Nonlinearity 3 (1990), 1015-1039.

[10] A. Gasull and J. Torregrosa, Samll-Amplitude limit cycles in Liénard systems via multiplicity, J. Diff. Eqs. 159 (1998), 1015-1039.

[11] I.S. Gradshteyn And I.M. Ryzhik, Table of Integrals, Series and Products, Academic Press, 1979.

[12] Y. Ilyashenko, Centennial history of Hilbert's 16th problem, Bull. Amer. Math. Soc. 39 (2002), 301-354.

[13] Chegnzhi Li And J. Llibre, Uniqueness of limit cycle for Liénard equations of degree four, submitted, 2010.

[14] Chegnzhi Li And J. Llibre, Liénard differential equations of degree 4 have at most one limit cycle, preprint, 2011.

[15] Jibin Li, Hilbert's 16th problem and bifurcations of planar polynomial vector fields, Internat. J. Bifur. Chaos Appl. Sci. Engrg. 13 (2003), 47-106.

[16] A. LiÉnARD, Étude des oscillations entrenues, Revue Génerale de l' Électricité 23 (1928), 946-954.

[17] A. Lins, W. De Melo And C.C. Pugh, On Liénard's Equation, Lecture Notes in Math 597, Springer, Berlin, (1977), pp. 335-357.

[18] J. LliBRE, Averaging theory and limit cycles for quadratic systems, Radovi Matematicki 11 (2002), 215-228.

[19] J. Llibre, A.C. Mereu and M.A. Teixeira, Limit cycles of the generalized polynomial Liénard differential equations, Math. Proceed. Camb. Phyl. Soc. 148 (2009), 363-383.

[20] N.G. Lloyd, Limit cycles of polynomial systems-some recent developments, London Math. Soc. Lecture Note Ser.,127, Cambridge University Press, 1988,pp. 192-234.

[21] N. G. Lloyd And S. Lynch, Small-amplitude limit cycles of certain Liénard systems, Proc. Royal Soc. London Ser. A 418 (1988), 199-208.

[22] N. Lloyd and J. Pearson, Symmetric in planar dynamical systems, J. Symb. Comput. 33 (2002), 357-366.

[23] S. LYNCH, Limit cycles if generalized Liénard equations, Applied Math. Letters 8 (1995), $15-17$,

[24] S. LyNCH, Generalized quadratic Liénard equations, Applied Math. Letters 11 (1998), 7-10,

[25] S. LyNCH, Generalized cubic Liénard equations, Applied Math. Letters 12 (1999), 1-6,

[26] S. LYNCH AND C. J. CHRISTOPHER, Limit cycles in highly non-linear differential equations, J. Sound Vib. 224 (1999), 505-517

[27] G.S. Rychkov, The maximum number of limit cycle of the system $\dot{x}=y-a_{1} x^{3}-a_{2} x^{5}, \dot{y}=$ $-x$ is two, Differential'nye Uravneniya 11 (1975), 380-391. 
[28] S. Smale, Mathematical Problems for the Next Century, Mathematical Intelligencer 20 (1998), $7-15$.

[29] F. Verhulst, Nonlinear Differential Equations and dynamical Systems, Universitext, Springer, 1991.

[30] P. Yu AND M. Han, Limit cycles in generalized Liénard systems, Chaos, Solitons and Fractals 30 (2006), 1048-1068.

1 División Académica de Ciencias Básicas, Ujat, Km 1 Carretera Cunduacán-Jalpa De MÉndez, C.P. 86690 Cunduacán Tabasco, MÉxico

E-mail address: justino.alavez@dacb.ujat.mx, gble@ujat.mx, jorge.lopez@dacb.ujat.mx

2 Departament de Matemàtiques, Universitat Autònoma de Barcelona, 08193 Bellaterra, Barcelona, Catalonia, Spain

E-mail address: jllibre@mat.uab.cat 